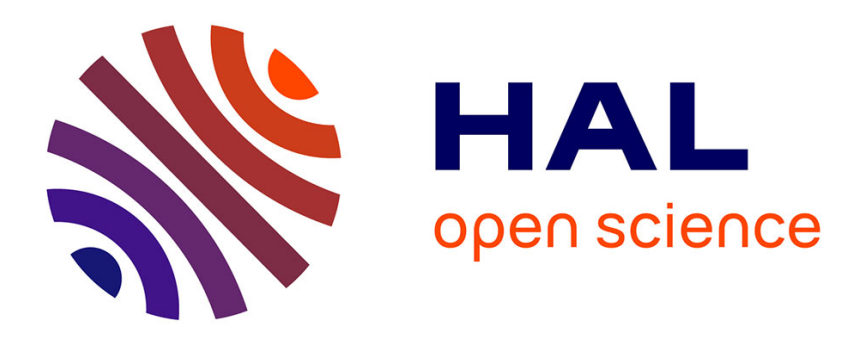

\title{
A Comparative Study on Multivariate Mathematical Morphology
}

\author{
Erchan Aptoula, Sébastien Lefèvre
}

\section{To cite this version:}

Erchan Aptoula, Sébastien Lefèvre. A Comparative Study on Multivariate Mathematical Morphology.

Pattern Recognition, 2007, 40 (11), pp.2914-2929. 10.1016/j.patcog.2007.02.004 . hal-00512660

\section{HAL Id: hal-00512660 \\ https://hal.science/hal-00512660}

Submitted on 31 Aug 2010

HAL is a multi-disciplinary open access archive for the deposit and dissemination of scientific research documents, whether they are published or not. The documents may come from teaching and research institutions in France or abroad, or from public or private research centers.
L'archive ouverte pluridisciplinaire HAL, est destinée au dépôt et à la diffusion de documents scientifiques de niveau recherche, publiés ou non, émanant des établissements d'enseignement et de recherche français ou étrangers, des laboratoires publics ou privés. 


\title{
A comparative study on multivariate mathematical morphology
}

\author{
E. Aptoula*, S. Lefèvre \\ Image Sciences, Computer Sciences and Remote Sensing Laboratory, \\ LSIIT-UMR-7005-CNRS Louis Pasteur University, \\ Pôle API, Blvd Sébastien Brant, PO Box 10413, 67412 Illkirch Cedex, France
}

\begin{abstract}
The successful application of univariate morphological operators on several domains, along with the increasing need for processing the plethora of available multivalued images, have been the main motives behind the efforts concentrated on extending the mathematical morphology framework to multivariate data. The few theoretical requirements of this extension, consisting primarily of a ranking scheme as well as extrema operators for vectorial data, have led to numerous suggestions with diverse properties. However, none of them has yet been widely accepted. Furthermore, the comparison research work in the current literature, evaluating the results obtained from these approaches, is either outdated or limited to a particular application domain. In this paper, a comprehensive review of the proposed multivariate morphological frameworks is provided. In particular, they are examined mainly with respect to their data ordering methodologies. Additionally, the results of a brief series of illustrative application oriented tests of selected vector orderings on colour and multispectral remote sensing data are also discussed.
\end{abstract}

Key words: Multivariate mathematical morphology, vector ordering, vectorial processing, colour images, multispectral images

\section{Introduction}

The mathematical morphology (MM) theory, founded by G. Matheron [57] and J. Serra [75,76], is a powerful image analysis framework, nowadays fully developed for both binary and greyscale images. Its popularity in the image

* Corresponding author. Tel: +33(0)39024 44 99; fax: +33(0)390 244455

Email address: aptoula@lsiit.u-strasbg.fr (E. Aptoula). 
processing community is mainly due to its rigorous mathematical foundation as well as its inherent ability to exploit the spatial relationships of pixels. The morphological framework provides a rich set of tools able to perform from the simplest to the most demanding tasks: noise reduction, edge detection, segmentation, texture and shape analysis, etc. As a methodology, it has been applied to almost all application areas dealing with digital image processing [78]. Consequently, it was only a matter of time before attempting to extend the same concepts to colour and more generally multivalued images.

Unfortunately, this extension is not straightforward. Specifically, the morphological framework is based on complete lattices, thus in order to accommodate multivalued images, a way of calculating the extrema of vectorial data is essential. Yet unlike scalars, there is no unambiguous way of ordering vectors. And besides, most of the known vector ordering schemes have already been employed for defining multivariate morphological operators. However, none of them has yet been widely accepted.

A few surveys on the different approaches have appeared occasionally in the literature [26,33]; although of excellent quality, they have become nevertheless relatively outdated and miss the newest developments. In the light of these remarks, the primary contribution of this paper is to provide an up to date overview of the existing approaches used for extending the fundamental operators of MM to multivariate data; and that is why cases limited only to specific operators have been omitted (e.g. colour image segmentation). Due to their rich diversity and overwhelming number, they are examined according to their methodology of ordering their multivalued input.

The secondary contribution of our work, differentiating it from previous surveys on this topic, is in terms of an application oriented comparative study of the major approaches. More precisely, the available comparison research work, is restricted either to a particular application (e.g. noise reduction) or image type (e.g. colour images) or vector space $[26,28,62]$. In this paper however, for the sake of objectivity, we provide the results of three types of tests realised with two kinds of multivalued data. In particular, we used colour images for noise reduction and texture classification, as well as multispectral remote sensing image data for pixel classification. The tests were conducted with the end of both comparing the suitability of different vector ordering schemes as basis for morphological operators, and for asserting their theoretical properties with the help of experimental results.

The organisation of the paper is as follows. The next section introduces the relevant fundamentals of extending morphological operators to multivariate images, whereas section 3 presents the current implementation variants of multivariate morphological frameworks. A discussion on the results of a series of illustrative application oriented tests of selected vector orderings takes place 
in section 4; and finally section 5 is devoted to concluding remarks.

\section{Extension of mathematical morphology to multivariate data}

In this section, we recall briefly the theoretical concepts behind the extension of morphological operators to multivariate images. For an in-depth study of the theory behind multivariate mathematical morphology the reader can refer to $[33,77]$.

\subsection{Orderings}

As the concept of order plays a central role in this paper, we start by recalling the relative definitions. A binary relation $\mathcal{R}$ on a set $\mathcal{S}$ is called:

- reflexive if $x \mathcal{R} x, \forall x \in \mathcal{S}$

- anti-symmetric if $x \mathcal{R} y$ and $y \mathcal{R} x \Rightarrow x=y, \forall x, y \in \mathcal{S}$

- transitive if $x \mathcal{R} y$ and $y \mathcal{R} w \Rightarrow x \mathcal{R} w, \forall x, y, w \in \mathcal{S}$

- total if $x \mathcal{R} y$ or $y \mathcal{R} x, \forall x, y \in \mathcal{S}$

A binary relation $<$ that is reflexive and transitive is called a pre-ordering (or quasi-ordering); if the anti-symmetry constraint is also met, it becomes an ordering. If additionally the totality statement holds for $<$, it is denoted as total, if not partial.

\subsection{Complete lattices and multivariate morphology}

The complete lattice theory is widely accepted as the appropriate algebraic basis for mathematical morphology. Besides unifying the approaches previously employed in binary and greyscale morphology, it also makes it possible to generalise the fundamental concepts of morphological operators to a wider variety of image types and situations. Extensive details on the lattice based description of the MM theory can be found in $[41,43,71,73,76,77]$. As a remark, we should also add that the scope of MM has also been extended to complete semilattices, that are more general than complete lattices $[42,45,46]$.

Specifically, a complete lattice $\mathcal{L}$ is a non empty set equipped with a partial ordering $<$, such that every non-empty subset $\mathcal{P}$ of $\mathcal{L}$ has a greatest lower bound $\wedge \mathcal{P}$, called infimum, and a least upper bound $\bigvee \mathcal{P}$, called supremum. In this context, images are modelled by functions mapping their domain space $\mathcal{E}$, an arbitrary non empty set that is an abelian group with respect to + , into 
a complete lattice $\mathcal{T}$, defining the set of possible "grey values". Moreover, if $\mathcal{F}$ represents the set of functions $f: \mathcal{E} \rightarrow \mathcal{T}$, then for the partial ordering:

$$
f, g: \mathcal{E} \rightarrow \mathcal{T}, f<g \Leftrightarrow \forall x \in \mathcal{E}, \quad f(x)<g(x)
$$

$\mathcal{F}$ also forms a complete lattice, where " $f(x)<g(x)$ " refers to the partial ordering in $\mathcal{T}$. In other words a complete lattice structure is imposed on the pixel intensity range. Usually $\mathcal{E}$ (the space of pixels) is taken to be either $\mathbb{R}^{d}$ (d-dimensional Euclidean space) or $\mathbb{Z}^{d}$ (d-dimensional discrete space), hence $\mathcal{F}$ corresponds respectively to the set of continuous or discrete images. Likewise various choices are available for $\mathcal{T}$, such as $\mathcal{T}=\overline{\mathbb{R}}^{n}$ and $\mathcal{T}=\overline{\mathbb{Z}}^{n}$. The case of $n>1$ corresponds to the so-called multivalued images [32]. Namely, in the case of a multivalued image with $n$ components, $\mathcal{T}=\mathcal{T}_{1} \times \ldots \times \mathcal{T}_{n}$ is considered as the cartesian product of $n$ complete lattices, and each mapping $f_{i}: \mathcal{E} \rightarrow \mathcal{T}_{i}, \quad i \in\{1, \ldots, n\}$ is called a channel or band of the multivalued image.

Within this model, morphological operators are represented by mappings between complete lattices (i.e. the input and output images) with some additional properties such as increasingness and translation invariance. They are employed in combination with matching patterns, called structuring elements (SE), that are usually subsets of $\mathcal{E}$ (i.e. flat SE). Particularly, erosion and dilation constitute the fundamental blocks of MM, from the combinations of which several sophisticated operators can be derived. More precisely, given two complete lattices $\mathcal{L}$ and $\mathcal{M}$, from an algebraic point of view, an operator $\varepsilon: \mathcal{L} \rightarrow \mathcal{M}$ is called an erosion, if it is distributive over infima, i.e. $\varepsilon\left(\bigwedge_{i} P_{i}\right)=\bigwedge_{i} \varepsilon\left(P_{i}\right)$ for every collection $\left\{P_{i}\right\}$ of elements of $\mathcal{L}$. Similarly, $\delta: \mathcal{L} \rightarrow \mathcal{M}$ is called a dilation, if it is distributive over suprema, i.e. $\delta\left(\bigvee_{i} P_{i}\right)=\bigvee_{i} \delta\left(P_{i}\right)$ for every collection $\left\{P_{i}\right\}$ of elements of $\mathcal{L}$. As suggested in [75], dilation and erosion basically rely on three concepts: a ranking scheme, the extrema derived from this ranking and finally the possibility of admitting an infinity of operands. Yet, the first two are missing from multivalued images.

For example, if we apply the preceding notions to the case of continuous multidimensional greyscale images $\left(f: \mathbb{R}^{d} \rightarrow \mathbb{R}\right)$, it suffices to replace the partial ordering $<$ of $\mathcal{T}$ with the usual comparison operator in $\mathbb{R}$, in order to induce a complete lattice structure on $\mathcal{T}$ and subsequently on $\mathcal{F}$ by means of (1), which will make the computation of extrema possible during erosion and dilation. Likewise, the inclusion operator " $\subset$ " can be used with binary images $\left(f: \mathbb{R}^{d} \rightarrow\{0,1\}\right)$. However, if we now consider multivalued images $\left(f: \mathbb{R}^{d} \rightarrow \mathbb{R}^{n}, n>1\right.$ ), it becomes problematic to find an ordering relation for the vectors of $\mathbb{R}^{n}$, due to the fact that there is no universal method for ordering multivariate data.

In order to remedy this inconvenience, in the classic paper of Goutsias et al. [33] it is proposed to employ an adequate surjective mapping $h$ to transform 
the image data into a more "suitable" space for morphological operators. More precisely, the idea of using a surjective mapping $h: \mathcal{T} \rightarrow \mathcal{L}$, where $\mathcal{T}$ is a non empty set and $\mathcal{L}$ a complete lattice, constitutes the theoretical support upon which several of the present multivariate morphological frameworks are based. Specifically, its importance lies in the fact that $\mathcal{T}$ is no longer required to be a complete lattice, since the ordering of $\mathcal{L}$ can be induced upon $\mathcal{T}$ by means of $h$ :

$$
\forall t, t^{\prime} \in \mathcal{T}, \quad t \leq_{h} t^{\prime} \Leftrightarrow h(t) \leq h\left(t^{\prime}\right)
$$

hence making it possible to construct $h$-morphological operators on $\mathcal{T}$. Consequently, one can deal with multivalued images, $f: \mathcal{E} \rightarrow \mathbb{R}^{n}$, through the use of a well chosen mapping $h: \mathbb{R}^{n} \rightarrow \mathcal{L}$, where $\mathcal{L}$ is a new, more suitable space for lattice based operations $[32,33]$.

Besides, given an adequate vector ranking scheme, the vectorial erosion $\left(\varepsilon_{b}\right)$ and dilation $\left(\boldsymbol{\delta}_{b}\right)$ of a multivalued image $\mathbf{f}$ by a flat SE $b$, can be expressed immediately by means of the vectorial extrema operators $\sup _{v}$ and inf $f_{v}$ based on the given ordering:

$$
\begin{aligned}
& \varepsilon_{b}(\mathbf{f})(\mathbf{x})=\inf _{\mathbf{s} \in b}\{\mathbf{f}(\mathbf{x}+\mathbf{s})\} \\
& \boldsymbol{\delta}_{b}(\mathbf{f})(\mathbf{x})=\sup _{\mathbf{s} \in b}\{\mathbf{f}(\mathbf{x}-\mathbf{s})\}
\end{aligned}
$$

Therefore, the main obstacle preventing the extension of morphological operators to multivalued images, consists in defining an ordering relation that will induce a complete lattice structure on the set of vectorial pixel intensities.

\subsection{Vector orderings}

Especially in the last few decades, a lot of effort has been put in engineering a way of ordering vectors. Although numerous techniques for ordering multivariate data can be found in the literature [40,54,67,83,86,87], according to the classical paper of Barnett (1976) [12], they can be classified into one of the following groups.

Marginal ordering (M-ordering): Which corresponds to univariate orderings realised on every component of the given vectors:

$$
\forall \mathbf{v}, \mathbf{v}^{\prime} \in \mathbb{R}^{n}, \mathbf{v} \leq \mathbf{v}^{\prime} \Leftrightarrow \forall i \in\{1, \ldots, n\}, \quad v_{i} \leq v_{i}^{\prime}
$$

Data is ordered along each one of its channels independently from others. Hence also the name componentwise ordering.

Conditional (sequential) ordering (C-ordering): In which vectors are ordered by means of some of their marginal components, selected sequentially 
according to different conditions. Whereas the components not participating in the comparison process are listed according to the position of their ranked counterparts. Hence, the ordering of the vectors is conditioned upon the particular marginal set of ranked components. Lexicographical ordering constitutes a widely known example of C-ordering employing potentially all the available components of the given vectors:

$$
\forall \mathbf{v}, \mathbf{v}^{\prime} \in \mathbb{R}^{n}, \quad \mathbf{v} \leq_{L} \mathbf{v}^{\prime} \Leftrightarrow \exists i \in\{1, \ldots, n\}, \quad\left(\forall j<i, v_{j}=v_{j}^{\prime}\right) \wedge\left(v_{i} \leq v_{i}^{\prime}\right)
$$

But of course one can also restrict the comparison process to use only a subset of the available components, as in [40]. C-orderings are most suitable for cases where one can establish a priority among the image channels.

Partial ordering (P-ordering): In this case, "partial" is an abuse of terminology (section 2.1), since there are total orderings belonging to this particular class (section 3.3). That is why we will use the term P-ordering. P-orderings consist simply of pre-orderings that partition the given vectors into groups of equivalence with respect to order, rank or extremeness [84]. They are generally geometric in nature and account well for the inter-relations between components.

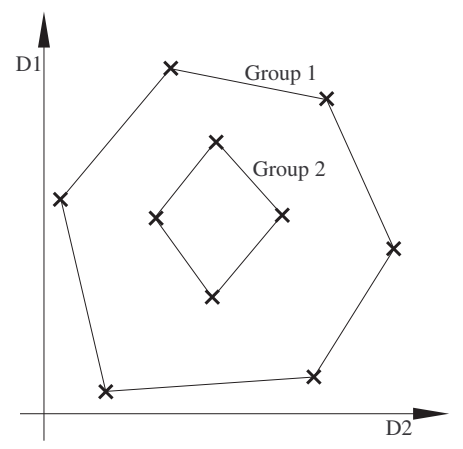

Fig. 1. A P-ordering example in a bi-dimensional space, based on the "peeling" principle.

A simple example of P-ordering is the one based on the "peeling" of a multivariate sample (figure 1). First the convex hull of the entire sample is calculated. Consequently, the points on the border of the convex hull constitute the first group. The points belonging to the border of the convex hull of the interior constitute the second, and so forth. As a result, the entire sample becomes partitioned and ranked according to their group number, but of course no internal distinction is in place for the contents of the groups.

Reduced ordering (R-ordering): In which vectors are first reduced to scalar values and then ranked according to their natural scalar order. A further categorisation of R-orderings consists in classifying them as distance orderings and projection orderings [56]. For instance, a R-ordering on $\mathbb{R}^{n}$ could consist in defining first a transformation $h: \mathbb{R}^{n} \rightarrow \mathbb{R}$, and then ordering the vectors 
of $\mathbb{R}^{n}$ with respect to the scalar order of their projection on $\mathbb{R}$ by $h$ :

$$
\forall \mathbf{v}, \mathbf{v}^{\prime} \in \mathbb{R}^{n}, \mathbf{v} \leq \mathbf{v}^{\prime} \Leftrightarrow h(\mathbf{v}) \leq h\left(\mathbf{v}^{\prime}\right)
$$

According to the chosen transformation it is possible to obtain a total preordering ( $h$ non-injective) or even a total ordering ( $h$ injective) [21]. An additional advantage of $\mathrm{R}$-orderings lies in the fact that with an adequately chosen $h$, they can attribute equal priority to all components, unlike C-orderings.

As the aforementioned ordering groups are not mutually exclusive, their combinations as well as numerous implementational variants have led to several morphological frameworks with diverse properties. The next section will elaborate on the different approaches.

\section{Approaches to multivariate mathematical morphology}

Despite the rich variety of multivariate morphological frameworks, there are in fact two main variables that are modified at each case, the extrema calculation method and the transformation (e.g. domain space change, etc) if any, that takes place on the image data before ranking; undoubtedly both influence the properties of the resulting operators.

In this section, we review in relative detail the approaches used for implementing multivariate morphological operators, primarily according to their vector ordering scheme, along with additional comments on their use with different preprocessing methods. However, instead of the conventional ordering categorisation presented in section 2.3, it was decided to adopt the scheme employed by Chanussot in [21] where they are classified according to their algebraic properties, as given in section 2.1. This choice was made with the aim of underlining the effect that these basic properties have on the end result. Additionally, the vectors of the ordering relations that are mentioned in the sequel, are considered in $\mathbb{R}^{n}$, unless otherwise specified.

\subsection{Processing strategies}

Given a multivalued image, in practice there are two general methods of morphological processing: marginal (or componentwise/scalar) and vectorial.

Marginal Processing: It consists in processing separately each channel of the image. The inter-channel correlation is totally ignored, along with all information that could be potentially used in order to improve the quality of 

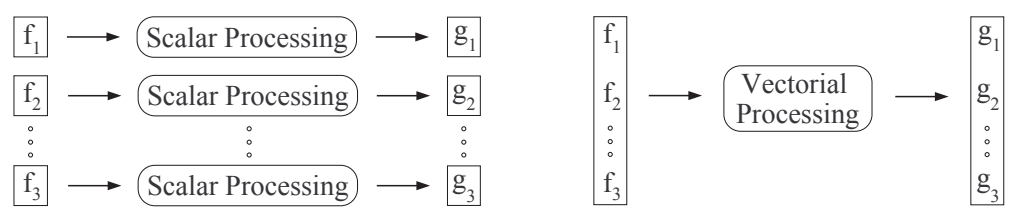

Fig. 2. Marginal (left) and vectorial processing (right) strategies

the result. Furthermore, the repetition of the processing procedure for each channel renders it expensive in terms of computational complexity. On the other hand, the marginal approach makes it possible to employ directly all methods offered by greyscale morphology (fig. 2 left).

Vectorial Processing: This main alternative of the marginal approach, as its name implies, processes all available channels globally and simultaneously. Given that the vectorial pixels are considered as the new processing units, the correlation among the different channels is no longer ignored (fig. 2 right). However, when compared to its marginal counterpart, the most important inconvenience of the vectorial approach appears to be primarily the need for adapting the existing algorithms in order to accommodate vectorial data; thus leading often to slower implementations than their scalar versions. The rest of this section elaborates on each approach as well as on their variations.

\subsection{Partial ordering based approach (Marginal)}

The so-called marginal processing strategy, despite being presented usually as an alternative to vectorial, is as a matter of fact no more than just its variation, as it employs the partial ordering defined in equation (5). Obviously, there can be vectors that may not be comparable under this ordering relation, for instance $\mathbf{a}=[7,2]^{T}$ and $\mathbf{b}=[3,4]^{T}$. Nevertheless, this does not prevent the definition of valid morphological operators based on extrema computed by means of this ordering [77]. Furthermore, it makes it possible to employ all tools offered by greyscale morphology with no need for special adaptation steps. For instance, the erosion and dilation expressions given in section 2.2 become equivalent to:

$$
\begin{aligned}
\boldsymbol{\varepsilon}_{b}(\mathbf{f})(\mathbf{x}) & =\left[\varepsilon_{b}\left(f_{1}\right)(\mathbf{x}), \ldots, \varepsilon_{b}\left(f_{n}\right)(\mathbf{x})\right]^{T} \\
\boldsymbol{\delta}_{b}(\mathbf{f})(\mathbf{x}) & =\left[\delta_{b}\left(f_{1}\right)(\mathbf{x}), \ldots, \delta_{b}\left(f_{n}\right)(\mathbf{x})\right]^{T}
\end{aligned}
$$

where $\varepsilon_{b}$ and $\delta_{b}$ denote respectively the scalar erosion and dilation operators with a SE $b$. For instance, Gu in [34] has employed the marginal approach for establishing multivalued morphological operators applied to moving object segmentation and tracking. Another example is given by Aptoula et al. in 
[10], where marginal morphological operators are employed with the purpose of galaxy detection from multispectral data. Moreover, a generalisation of the marginal approach was given by the matrix morphology theory of Wilson [97], which was based on the work of Heijmans and Ronse [43].

Despite its implementational simplicity, marginal ordering suffers mainly from two disadvantages: not accounting for inter-channel information as well as the risk of altering the spectral composition of its input. More precisely, as each component is processed independently, any eventual correlation among them is totally ignored, hence rendering this approach unsuitable for images with highly correlated components (e.g. RGB colour images) [11]. A possible solution to this problem, as proposed in [33], consists in applying a decorrelating transformation (e.g. maximum noise fraction transform (MNF), principal component analysis (PCA), discrete cosine transform, a proper colour space, etc) prior to ordering.

An application of this idea to the detection of land mines, in combination with the MNF transform, can be found in [13]. Or in [14], where hyperspectral remote sensing images are classified by means of morphological profiles computed marginally from the first two principal components of the input. Nevertheless, these decorrelating transformations also introduce an additional computational burden.

Furthermore, there is absolutely no guarantee that marginally processed vectors belong to the input image. The lack of vector preservation constitutes an undesirable effect for several applications. For example, in the case of colour image processing this would lead to the appearance of new colours (also known as false colours) and thus deteriorate the visual quality of the result, and in particular the colour balance and the object boundaries; whereas the effect near the spatial edges, leads to the so-called edge jitter. Similarly, it can be harmful for the spectral signature based classification of remote sensing images, where an altered spectral composition can damage the entire process.

A detailed study of the problem of vector preservation can be found in $[44,82]$. According to the last reference, the only way to use morphological operators without generating new vectors is to impose an order on the vector space by means of an (pre-)ordering verifying the totality constraint; besides, this is why the majority of the published articles on multivalued morphology deal with total (pre-)orderings. Yet, vector preserving approaches are in the same time limited by this property, due to the restriction imposed on their output (i.e. the output must be a vector from the input set); whereas the marginal approach has access to a much broader range of output values, an advantage which becomes most valuable during noise reduction [26].

Of course, there are also situations such as the one presented in [85], where the 
inter-channel correlation level is low (near and mid infra-red bands) and vector preservation is not a necessity; in which case the marginal operators used for detecting the dim point targets on maritime backgrounds perform adequately. Additional attempts motivated by the intuitiveness of the marginal approach have resulted in improvements. Namely, Serra in [77] presents an intermediate form between a M-ordering and a C-ordering, while an application of marginal ordering in the HSV colour space is introduced by Weber and Acton in [95], where the aforementioned undesirable effects are reduced by shifting the hue origin. Moreover, Al-Otum in [1], proposes the corrected componentwise morphological algorithm with the end of preventing the appearance of new vectors. It consists in replacing each new vector of the output with its closest vector from the original image, chosen with the help of a Mahalanobis distance based error function.

In brief, either accompanied by additional transformations or not, the marginal processing strategy uses conventional greyscale operators and pixels are still treated as scalar values.

\subsection{Total pre-ordering based approach}

Contrarily to the marginal approach, by means of the additional property of totality, all vectors become comparable and as a result it is possible to construct a totally ordered lattice structure. Hence pixels can be manipulated as whole vectors, and consequently the risk of altering the pixel composition of the input is eliminated [82].

All pre-orderings however share a common drawback, which is the relaxation of the anti-symmetry constraint. Thus distinct vectors can eventually end up being equivalent. That is why additional measures become necessary, in order to resolve the ambiguity of eventually multiple extrema. For instance a partial, but considerably useful in practice solution proposed by Comer and Delp in $[25,26]$ against this problem, consists in selecting the output vector according to its position in the SE.

Reduced total pre-orderings: Total pre-orderings can be obtained with Rorderings employing a non-injective reduction transformation [21]. Distance measures are typical examples of such transformations; in fact they account for the majority of the proposed R-orderings.

This first variant, ranks a family $\left\{\mathbf{v}_{j}\right\}$ of vectors according to their distance from a reference vector $\mathbf{v}_{r e f}$ :

$$
\forall \mathbf{v}_{k}, \mathbf{v}_{l} \in\left\{\mathbf{v}_{j}\right\}, \quad \mathbf{v}_{k} \leq \mathbf{v}_{l} \Leftrightarrow d\left(\mathbf{v}_{k}, \mathbf{v}_{r e f}\right) \leq d\left(\mathbf{v}_{l}, \mathbf{v}_{r e f}\right)
$$


where $d(\cdot, \cdot)$ represents a distance measure. An example of this type of Rordering, as applied to colour images, can be found in [74]. In case the reference vector is the origin, expression (10) becomes equivalent to using the norms of the vectors. A variation of expression (10), which eliminates the need for a reference vector, consists in associating each vector with the sum of its distances from the other vectors:

$$
\forall \mathbf{v}_{k}, \mathbf{v}_{l} \in\left\{\mathbf{v}_{j}\right\}, \quad \mathbf{v}_{k} \leq \mathbf{v}_{l} \Leftrightarrow \sum_{j} d\left(\mathbf{v}_{k}, \mathbf{v}_{j}\right) \leq \sum_{j} d\left(\mathbf{v}_{l}, \mathbf{v}_{j}\right)
$$

This additional advantage however comes at a high cost, in terms of both computational complexity and theoretical properties. Specifically, although this approach can be used for computing multivariate extrema, it does not constitute an ordering in the sense of a binary relation, hence leading to pseudo-morphological operators. Furthermore, the infimum of a family of vectors calculated in this way, corresponds to the notion of "median vector" and consequently does not carry the significance of a "minimum" in the numerical sense. As a remark, we should also add that in situations where the directions of vectors gain more importance than their magnitude, (10) and (11) may also be used by replacing the distance $d$ with its angular counterpart, as proposed in $[88]$.

There is also another property worth mentioning, referenced in [89], concerning (11) and implementations of (10), if the reference vector is chosen to be "in the middle" (e.g. the median or the average). It concerns the instability of the supremum computed by means of these orderings. Despite the remarkable stability of the infimum under the same conditions, even a slightly varied input can radically change the least upper bound. Consequently, the dilation operator that makes use of the supremum becomes unsound from a practical point of view.

The choice of the distance measure is of course another key topic. Theoretically, any kind of metric can be used, and so it is in practice. For instance, in an application of (11) to vector area morphology in [29], the city-block and Euclidean distances are considered. Whereas, in [68] the same reduction scheme is employed in combination with spectral angle distances to the end of creating morphological profiles for hyperspectral images. Moreover, Al-Otum $[1,90]$ uses $(10)$ coupled with the Mahalanobis distance for ordering the components of colour images in different colour spaces, with "black" being the reference vector. An example of Euclidean norm based ordering can be found in [26], as applied to RGB colour images.

Moreover, an original approach implemented in the polar version of the C-Y colour space appears in [2], where a saturation based combination of the hue and intensity planes is proposed. Specifically, the vectorial pixels contained within the SE are reduced into scalars by means of a weighted combination 
of vector angle $\left(d_{a}\right)$ and Euclidean distances $\left(d_{E}\right)$ from the vector $\mathbf{v}_{c}$ at the centre of the SE:

$$
h(\mathbf{v})=w\left(\mathbf{v}, \mathbf{v}_{c}\right) \cdot d_{a}\left(\mathbf{v}, \mathbf{v}_{c}\right)+\left(1-w\left(\mathbf{v}, \mathbf{v}_{c}\right)\right) \cdot d_{E}\left(\mathbf{v}, \mathbf{v}_{c}\right)
$$

where $w(\cdot, \cdot)$ denotes the weight computed based on the saturation levels of the given vectorial pixels. The underlying idea is to favour hue differences $\left(d_{a}\right)$ when saturation levels are high, while intensity differences $\left(d_{E}\right)$ gain more importance with achromatic pixels.

Of course the non-injective transformation choices are by no means limited by these few variants. For instance, in [48], vectors are ordered based on the number of times they appear in the input image. Nevertheless, distance based R-orderings hold the potential of accounting for all dimensions without privileging any of them, a property which becomes particularly useful in case there is no predefined order of importance among the available channels (e.g. RGB colour images). Otherwise any transformation capable of realising the necessary reduction may be used. For example in [26], an additional R-ordering is presented, applied to RGB colour images, where vectors are reduced to scalars with the help of a linear weighted combination:

$$
h(\mathbf{v})=w_{1} \cdot v_{1}+\ldots+w_{n} \cdot v_{n}
$$

Hence making possible the arbitrary prioritisation of colour channels by means of the $w_{i} \in \mathbb{R}, \quad i \in\{1, \ldots, n\}$ coefficients. Finally, a more complicated ordering scheme takes place in [50], where a new R-ordering based on ordinal first principal component analysis is introduced and the derived operators are applied to edge detection on RGB colour images.

Conditional total pre-orderings: As previously mentioned in section 2.3 C-orderings restrict the ordering process to only one or more components of the given vectors, while the others are conditioned upon them. That is why C-orderings are suitable for situations where certain channels are more "privileged" than others. Besides, unless all vector components participate in the ordering process, the resulting C-ordering is bound to be a total preordering, thus sharing their aforementioned inconveniences. For example, in the case where only the first component is employed [40]:

$$
\mathbf{v} \leq \mathbf{v}^{\prime} \Leftrightarrow v_{1} \leq v_{1}^{\prime}
$$

Hence, the two distinct vectors $\mathbf{a}=[7,2]^{T}$ and $\mathbf{b}=[7,3]^{T}$ would be considered equivalent according to (14). The main problem of C-orderings concerns of course the choice of the ordered components. Obviously, ordering vectors along only some of their components is practically justifiable only if the given components represent sufficiently the vectors.

In the general case, this approach attributes far too much significance to the 
selected components, disregarding all others. While usually this constitutes a severe problem, there are cases where it becomes invaluable. For instance, when it is a priori known that certain channels are noise free. In fact, Corderings are almost always used in combination with a suitable domain space change, so that the data of interest, in its majority, will lie in only some of the channels.

An application example can be found in [92], where a C-ordering on the HSV colour space is employed, ignoring the hue component. In fact, this also constitutes a fine example of a combined ordering; more precisely, the result can also be considered as a P-ordering where the ordered groups contain the colours of equal value and saturation with no internal distinction whatsoever as hue is not taken into account. An application of the same ordering on colour image skeletonisation can be found in [3].

\subsection{Total ordering based approach}

Total orderings, from a theoretical point of view, have two main advantages that render them more suitable for vector ordering, as far as multivariate MM is concerned. First, thanks to their totality, they are vector preserving, and contrarily to pre-orderings, as they verify the anti-symmetry constraint the computed extrema are unique. That is why the majority of the attempts concentrated on extending morphological operators to multivalued images are based on total orderings. In particular, the lexicographical ordering (C-ordering) along with its variants is among the most implemented choices.

However, the uniqueness of extrema takes a serious toll, since the prioritisation of certain vector components becomes inevitable [21]. That is why they are almost always used in combination with a suitable domain transformation (e.g. HSV, L*a*b* PCA, etc) that will place the "interesting" part of the data in the first few channels. Nevertheless, as it will be subsequently presented some implementations tend to be more "symmetric" than others.

Lexicographical ordering: Lexicographical ordering, introduced in (6), is undoubtedly the most widely employed total ordering within this context. As a conditional ordering, it is most suitable to situations where an order of "importance" exists on the available channels, either inherently or artificially created by means of an appropriate transformation.

Figure 3 provides an example of the priority attributed to the first component during lexicographical ordering. More precisely, a vectorial dilation is applied on a RGB colour image (fig. 3, left) and as red is the head component (dark grey), it dominates visibly over green (light grey) (fig. 3, middle). Whereas if we permute the channels as GRB, the effect is reversed in favour of green (fig. 

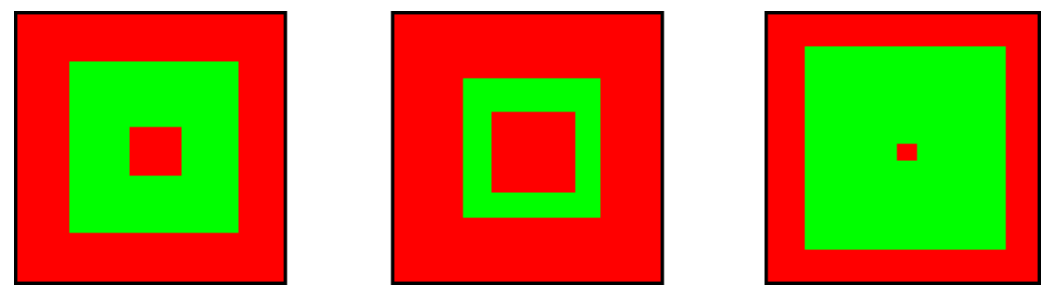

Fig. 3. original image (left), results of applying a vectorial dilation based on a lexicographical ordering (RGB - middle) (GRB - right) with a $21 \times 21$ square SE

3 , right).

Lexicographical ordering has been mostly employed in the domain of colour MM, where several colour spaces exhibit an inherent prioritisation among their channels as far as human observers are concerned. Specifically, as the human vision system is more sensitive to brightness changes than to chromatic changes, brightness components tend to be privileged [31]. Hue based spaces in particular have been extensively studied in the literature as they are closely related to the way which humans perceive colour. It should be additionally noted that the hue component poses considerable problems as it is a $2 \pi$ periodical value and thus cannot be intuitively ordered simply from smallest to largest $[38,66]$. An example of luminance based lexicographical ordering on the HSV colour space can be found in [53], whereas [51] and [91] use the same ordering respectively for median filtering and granulometry calculation on colour images. Of course there can also be specific situations where chromatic information is more significant, for instance in [60], hence hue is compared first in the lexicographical cascade.

Other applications of lexicographical ordering in the HSI space include [61], where reconstruction based morphological operators are developed with the end of brightness elimination, using an intensity based ordering scheme. The same principle is additionally employed in [63] with the purpose of noise elimination. The perceptually uniform $\mathrm{L}^{*} \mathrm{a}^{*} \mathrm{~b}^{*}[39]$ and $\mathrm{L}^{*} \mathrm{u}^{*} \mathrm{v}^{*}[66]$ spaces have also been used together with a lexicographical ordering. Moreover, a thorough study of the potential of this ordering in the HLS space is provided in [37]; while in [7], the use of lexicographical ordering in the improved HLS (IHLS) space is explored. And finally, the results of a comparative application of this ordering in different hue based colour spaces can be found in [62].

As the majority of lexicographical comparisons are determined by the first components [39], variations of the classical lexicographical ordering were proposed, with the end of better tuning the priority as well as degree of influence of each component. A non exhaustive list of such extensions follows. A first group of variations is based on the use of additional components during comparison. Rivest in $[69,70]$ has exploited the symmetrical nature of a norm based R-ordering by placing it to the first position of the lexicographical cascade, 
with the end of developing morphological operators for complex signals:

$$
\mathbf{v} \leq \mathbf{v}^{\prime} \Leftrightarrow\left[\|\mathbf{v}\|, v_{1}, \ldots, v_{n}\right]^{T} \leq_{L}\left[\left\|\mathbf{v}^{\prime}\right\|, v_{1}^{\prime}, \ldots, v_{n}^{\prime}\right]^{T}
$$

where $\leq_{L}$ is the lexicographical ordering. Similar variants to (15) have been proposed in $[5,74]$, with the difference of placing a reference vector based distance measure in the position of the first component. Of course, there is no limit to the number or type of functions that can be used according to this principle. Other examples include the use of the maximum and minimum of the compared components in the case of RGB colour images, as well as their weighted combinations [8]. Another type of extension to the classical lexicographical ordering consists in using of a user defined parameter $\alpha$ in such a way that it can modify the degree of influence of the first component. For instance, the $\alpha$-modulus lexicographical ordering, introduced by Angulo $[4,6]$ :

$$
\forall \mathbf{v}, \mathbf{v}^{\prime} \in \mathbb{Z}^{n}, \mathbf{v} \leq \mathbf{v}^{\prime} \Leftrightarrow\left[\left\lceil v_{1} / \alpha\right\rceil, v_{2}, \ldots, v_{n}\right]^{T} \leq_{L}\left[\left\lceil v_{1}^{\prime} / \alpha\right\rceil, v_{2}^{\prime}, \ldots, v_{n}^{\prime}\right]^{T}
$$

was implemented on a variation of the HLS colour space. Specifically, by dividing the first component with a parameter $\alpha$, and then rounding it off to the next closest integer, a sub-quantisation of the first vector dimension is realised, hence forming larger equality groups within this dimension. Consequently, a higher number of comparisons is expected to reach the second dimension.

Bit mixing based ordering: Bit interlacing (or mixing) constitutes an innovative R-ordering, aiming mainly to eliminate the unavoidable asymmetry, which results from the application of total orderings. Specifically it employs an injective transformation exploiting the binary representation of each component in order to impose a total order on the vector space $[21,22,24]$.

Given a vector $\mathbf{v}$, with each component coded in $k$ bits, the corresponding reduction transformation $h: \mathbb{Z}^{n} \rightarrow \mathbb{Z}$ is formulated as:

$$
h(\mathbf{v})=\sum_{m=1}^{k}\left\{2^{n \cdot(k-m)} \cdot \sum_{i=1}^{n} 2^{n-i} \cdot v_{i, m}\right\}
$$

where $v_{i, m}$ denotes the $m^{\text {th }}$ bit of the $i^{t h}$ component of $\mathbf{v}$. Hence, the resulting binary representation of $h(\mathbf{v})$ becomes:

$$
v_{1,1} v_{2,1} \ldots v_{n, 1} v_{1,2} v_{2,2} \ldots v_{n, 2} \ldots v_{1, k} v_{2, k} \ldots v_{n, k}
$$

Besides being endowed with all the qualities of a total ordering, bit mixing provides a more symmetrical approach than its lexicographical counterpart as 
dimensions are mixed in bit level. Of course some dimensions continue to be more privileged than others, with the degree of that privilege being proportional to the significance of the bit position that they occupy. Furthermore, a finer grained symmetry can be obtained by modifying the original mix order [21]. Then again, there are several situations where data channels need to be processed with a certain priority. Bit mixing can easily respond to this requirement by placing the important vector components to more significant bit positions. Additionally, bit interlacing can also be used as a means of fusing the channels, hence allowing for instance the use of the watershed transformation on colour images [23].

From a theoretical point of view, this approach aims to fill a given multidimensional space using a "balanced" space filling curve (SFC) with respect to the available dimensions. In the case of total orderings, these curves (e.g. Peano curve) pass through all vector coordinates of the space under consideration, hence vectors can be ordered according to their position on it. An additional approach developed based on SFC can be found in [81]. On the other hand, according to [80] the main inconvenience of a total ordering obtained in this fashion is its lack of physical interpretation.

\subsection{Other approaches}

Besides the previously presented ordering methodologies, there have been also some rather "unconventional" orderings or extremum calculation approaches, in the sense that they either do not consist in simply using a standard vector ordering scheme, or they are developed for a particular form of image data, or even combine additional theories with the end of achieving an efficient solution.

For example, following the relatively recent success of fuzzy morphology with greyscale images $[16,27]$, a couple of attempts to apply the fuzziness concepts to multivariate images have already been carried out. Köppen et al. in [47], introduced fuzzy Pareto morphology, a means of computing multivariate extrema based on the notion of Pareto sets, a concept belonging to the field of multicriteria optimisation, combined with fuzzy subsethood. However, since there is no underlying binary ordering relation, it results in pseudo-morphological operators. A second attempt was made by Louverdis et al. [52], who employ a direct fuzzification of the vectorial pixels in the HSV colour space, that are subsequently ranked according to a lexicographical ordering scheme. The morphological operators based on this total pre-ordering have been compared in the original article with their counterparts in [53] resulting from the use of a similar lexicographic ordering also in the HSV space. According to their experiments, the fuzzy version has slightly better noise reduction capabilities 
as well as less sensitivity to distortions.

Moreover, the domain of medical imaging has recently introduced matrixvalued images, where a positive definite matrix is associated to each voxel. Pioneering work on the application of MM theory to this type of images has been made by Burgeth et al. [17-19], using both analytic (i.e. Loewner ordering) and geometric approaches (i.e. modelling matrices with ellipsoids).

Some further original approaches aiming to compute multivariate extrema, include the one proposed in [96], developed with the purpose of colour object detection using vector projection measures in combination with vectorial SEs, as well as the graph based methodology introduced by Lezoray et al. [49], employing the minimum spanning tree algorithm among the pixels under the SE, which however leads to pseudo-morphological operators. An ordering based on labels has been additionally proposed by Ronse and Agnus [72]. Moreover, Zaharescu et al. have explored in [98] the potential of the triangle representation of colours, which leads to a R-ordering, while Mojsilovic and Soljanin [58] have employed a method based on a quantization using Fibonacci lattices, in order to obtain a partial ordering. And finally Gibson et al. [30] have relied on local convex hull computations for locating multivariate extrema, whereas Busch and Eberle [20] have proposed a conditional ordering based on semantic principles.

\subsection{Synopsis}

This section presented the different approaches that have appeared so far in the literature, with the end of extending morphological operators to multivalued images. A summary of the related references is given in table 1 .

\section{Experiment results}

Having provided an insight into several multivariate morphological frameworks, in this section a brief series of comparative tests will be carried out with the aim of measuring their relative performances in three image processing tasks.

So far, the comparative studies of multivalued morphological processing approaches $[26,28,62,93,94]$ have been restricted to a particular application domain (e.g. colour morphology) or vector space. The reason of these restrictions is the overwhelming number of crucial variables that need to be taken into account in order to achieve a fully objective performance measure (e.g. number 
Table 1

Synoptic table of references developing multivariate morphological operators, organised according to the properties of the employed ordering scheme and its corresponding implementation space (VP: vector preserving, PB: prioritisation of bands)

\begin{tabular}{|c|c|c|c|}
\hline \multirow{2}{*}{ Data space } & \multicolumn{3}{|c|}{ Properties } \\
\hline & $\neg \mathrm{VP}$ & $\mathrm{VP} \wedge \mathrm{PB}$ & $\mathrm{VP} \wedge \neg \mathrm{PB}$ \\
\hline Generic & {$[26,33,34,58]$} & {$[5,20,22,24,30,58,72]$} & {$[1,5,26,29,47-50,68,81,98]$} \\
\hline HSV & {$[95]$} & {$[3,35,51-53,60,91,92]$} & \\
\hline $\mathrm{L}^{*} \mathrm{a}^{*} \mathrm{~b}^{*}$ & & {$[39]$} & \\
\hline$L^{*} u^{*} v^{*}$ & & {$[66]$} & \\
\hline (I)HLS & & {$[6-8,37,96]$} & \\
\hline HSI & & {$[61,63,64]$} & \\
\hline $\mathrm{C}-\mathrm{Y}$ & & {$[2,74]$} & \\
\hline Complex & & {$[69,70]$} & {$[96]$} \\
\hline Matrix & & & {$[17-19]$} \\
\hline
\end{tabular}

of images, type of images, number of channels, correlation of channels, vector space transformations, etc). That is why the aim of this section is not to realise a "benchmark" of the different approaches, but rather to provide indications on their suitability for particular input properties, as well as to assert the remarks made in the previous sections with experimental results.

For the sake of simplicity only certain approaches have been considered during the tests, chosen subjectively as representative members of the classification given in section 2.1. More precisely, the ordering schemes participating in the tests are: marginal (M), bit mixing (B), lexicographical (L), a variation of lexicographical ordering $(\mathrm{rL})$, equation (15), reference vector based distance orderings using the median $(\mathrm{R})$ and the origin $(\mathrm{N})$ as reference vectors, as well as the method based on cumulative distances (C), although it does not qualify as an ordering from an algebraic point of view. The Euclidean distance and $L_{2}$ norm are used where necessary. As far as their implementation is concerned, vector comparisons were hard coded without the use of any indexes [82], within a generic Java based framework. The subjects are chosen from the two primary application areas of multivariate morphology, colour and remote sensing imagery. In particular, noise reduction and texture classification tests are realised with the first, while the latter are used for a pixel based classification. 


\subsection{Noise reduction}

During this first test, the relative performances of the vector ordering schemes are examined in terms of noise reduction quality. As a quantitative measure, the normalised mean squared error (NMSE) is used:

$$
\mathrm{NMSE}=\frac{\sum_{i=1}^{N} \sum_{j=1}^{M}\left\|\mathbf{f}(i, j)-\mathbf{f}^{\prime}(i, j)\right\|^{2}}{\sum_{i=1}^{N} \sum_{j=1}^{M}\|\mathbf{f}(i, j)\|^{2}}
$$

where $N$ and $M$ represent the image dimensions while $\mathbf{f}(i, j)$ and $\mathbf{f}^{\prime}(i, j)$ denote respectively the vectorial pixels at position $(i, j)$ for the original and filtered images. The tests have been repeated with a number of RGB colour images of various content, however here we exhibit the results obtained for the "Lenna" image (fig. 4). The subjects, of size $512 \times 512$ pixels and 24 bits per pixel, are first contaminated with zero-mean additive Gaussian noise, $\sigma=32$ and correlation factor $\rho=0.0$ (table 2) and $\rho=0.9$ (table 3). Other noise distributions that have also been tested, include double-exponential and uniform, however as the influence of the type of noise distribution on the resulting performances was observed to be minimal, they were omitted from tables 2 and 3 for the sake of clarity.
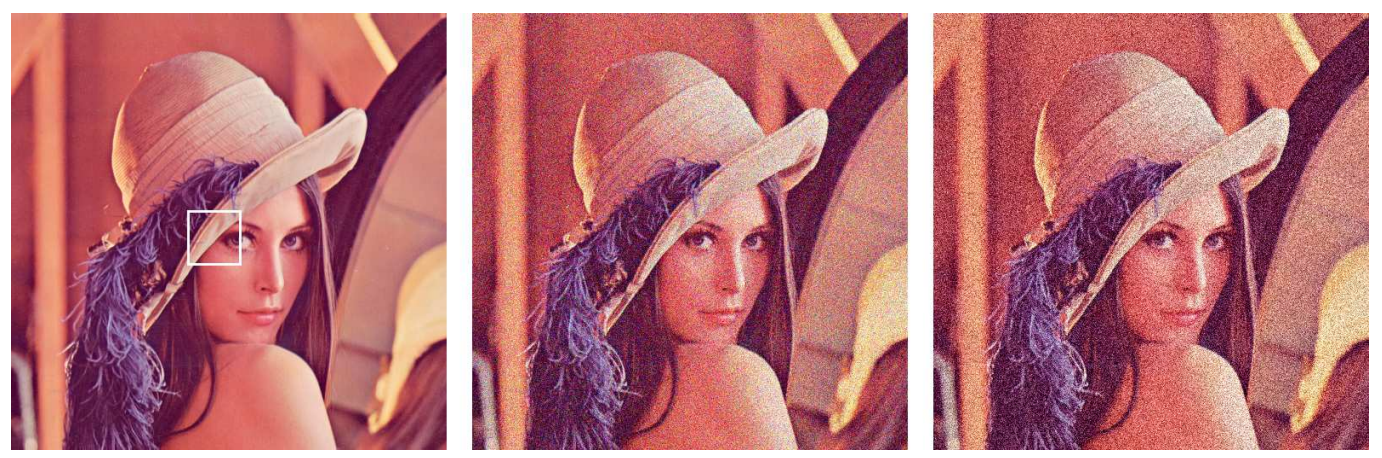

Fig. 4. From left to right: the original image "Lenna", corrupted with uncorrelated Gaussian noise $(\sigma=32, \rho=0.0)$ and corrupted with correlated Gaussian noise $(\sigma=32, \rho=0.9)$. The white square represents the enlarged results in figure 5

The filter employed for smoothing is open-close close-open (OCCO). More precisely, let first $\gamma_{b}$ and $\phi_{b}$ denote respectively the vectorial opening and closing operators:

$$
\begin{aligned}
& \gamma_{b}(\mathbf{f})(x, y)=\boldsymbol{\delta}_{b}\left(\varepsilon_{b}(\mathbf{f})\right)(x, y) \\
& \phi_{b}(\mathbf{f})(x, y)=\varepsilon_{b}\left(\boldsymbol{\delta}_{b}(\mathbf{f})\right)(x, y)
\end{aligned}
$$

In which case, OCCO is defined as the pixelwise average of open-close and 
close-open:

$$
\mathrm{OCCO}_{b}(\mathbf{f})(x, y)=\frac{1}{2} \boldsymbol{\gamma}_{b}\left(\boldsymbol{\phi}_{b}(\mathbf{f})\right)(x, y)+\frac{1}{2} \boldsymbol{\phi}_{b}\left(\boldsymbol{\gamma}_{b}(\mathbf{f})\right)(x, y)
$$

given the size of the input, the SE $b$ is chosen as a cross of size $3 \times 3$. Larger sizes have been also tested but the relative performances remained almost the same. The OCCO filter is chosen primarily due to its effective combination of the basic morphological operators as well as for its suitability against the chosen noise type. On the other hand, taking the average of the intermediate results, obviously prevents the preservation of the initial vectors. Nevertheless, this is a desired property in the context of noise reduction as it makes it possible to better approximate the original noise free values. Different colour spaces have been also employed during smoothing for mainly two reasons; first in order to simulate various correlation levels among the channels as well as for testing the effect that uneven distributions of the intensity information among the channels have on the underlying ordering schemes. Moreover, as hue based colour spaces usually require a reference value, they have been excluded from the experimentation process with the end of avoiding any further parametrisation.

Table 2

NMSE $\times 100$ values for the "Lenna" image obtained against uncorrelated Gaussian noise

\begin{tabular}{|c||c|c|c|c|c|c|c|}
\hline \multicolumn{1}{|c||}{$\begin{array}{l}\text { Colour } \\
\text { spaces }\end{array}$} & \multicolumn{7}{|c|}{ Vector Orderings } \\
\cline { 2 - 8 } & $\mathrm{M}$ & $\mathrm{L}$ & $\mathrm{N}$ & $\mathrm{B}$ & $\mathrm{C}$ & $\mathrm{R}$ & $\mathrm{rL}$ \\
\hline RGB & $\mathbf{0 . 7 8}$ & 2.29 & 2.10 & 2.21 & 3.10 & 3.07 & 2.10 \\
\hline GBR & $\mathbf{0 . 7 8}$ & 2.33 & 2.10 & 2.19 & 3.10 & 3.07 & 2.10 \\
\hline L*a*b* $^{*}$ & $\mathbf{0 . 9 2}$ & 2.23 & 2.50 & 2.05 & 3.13 & 3.02 & 2.50 \\
\hline YUV & $\mathbf{0 . 7 9}$ & 2.09 & 2.12 & 2.03 & 2.64 & 2.59 & 2.12 \\
\hline
\end{tabular}

Table 2 shows the NMSE results of the orderings within four colour spaces against uncorrelated Gaussian noise. The entries corresponding to the best performance of their row (colour space) are in bold. According to the obtained values, the overall superiority of the marginal approach over its vectorial counterparts is remarkable.

As stated earlier the reduced smoothing capacity of vectorial approaches is part of the trade-off between noise reduction capability and vector preservation. In brief, even with a maximized processing symmetry among the available channels the final result of vectorial openings and closings in equation (22) will necessarily be one of the input vectors. And as such it is natural for the marginal strategy to outperform, by having access to a much broader range of output values, that are not necessarily included however in the original image. 
A second remark concerns the relatively high error rates of the total orderings in the highly correlated RGB space, which is simply due to the fact that both bit mixing and lexicographical orderings inevitably prioritise the vector components. Consequently, the green and especially blue channels influence the outcome of vector comparisons much less that red, thus resulting in poor smoothing quality in the last two channels. The effect of prioritisation is particularly visible with lexicographical ordering. An exception to this remark is rL, which although total, results always in a NMSE value identical to norm based reduced ordering $(\mathrm{N})$, due to the vector norm that occupies the first position, as indicated in equation (15), during the lexicographical comparison of vectors. Therefore, as the vast majority of comparisons is decided by means of the first vector component, from a practical point view it appears to behave exactly as a norm based R-ordering.

As fas as the three distance based approaches are concerned (R, C, N), the norm based ordering takes the lead while its distance based counterparts provide quite unsatisfactory results. Thanks to the non-injective scalarisation that takes place, no distinction is made among the channels during comparison. This last important property becomes more evident within the GBR space, a permutation of RGB, where they achieve the exact same performances thus underlining their robustness against situations where it is not a priori known in which channel takes place the majority of the "important" information or even which channel is the most corrupted.

Furthermore, the $\mathrm{L}^{*} \mathrm{a}^{*} \mathrm{~b}^{*}$ colour space causes significant changes in the results as it is less correlated than RGB and the brightness information is moved exclusively to the first channel. As expected, the bit mixing and lexicographical orderings improve their smoothing rates substantially, since their prioritisation works this time in their favour by rendering the brightness dimension more "exploitable". It is also presumed that the finer grained symmetry of the vector components during comparison is what makes it possible for the bit mixing ordering to surpass its lexicographical counterpart. Nevertheless, the marginal ordering still provides the best results.

On the other hand, table 3 shows the NMSE results obtained against highly correlated Gaussian noise. Although the relative performances of vectorial approaches have stayed the same, this time they are clearly much closer to that of marginal processing.

As far as the computation times are concerned, table 4 illustrates the relative duration of a single application of the OCCO filter for each ordering. Apparently the lexicographical ordering is the fastest among them, closely followed by the marginal approach, while the R-orderings suffer from the extensive distance calculations. 

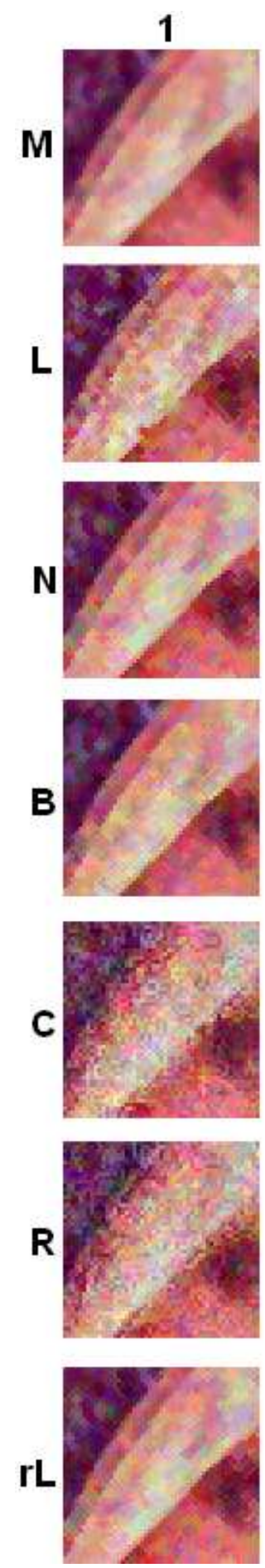
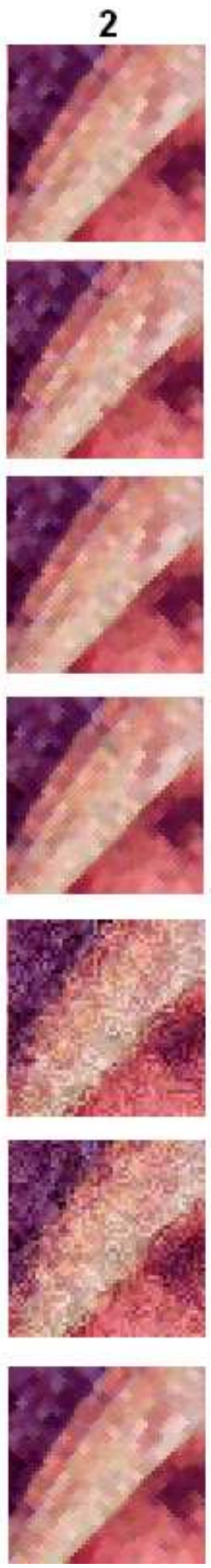
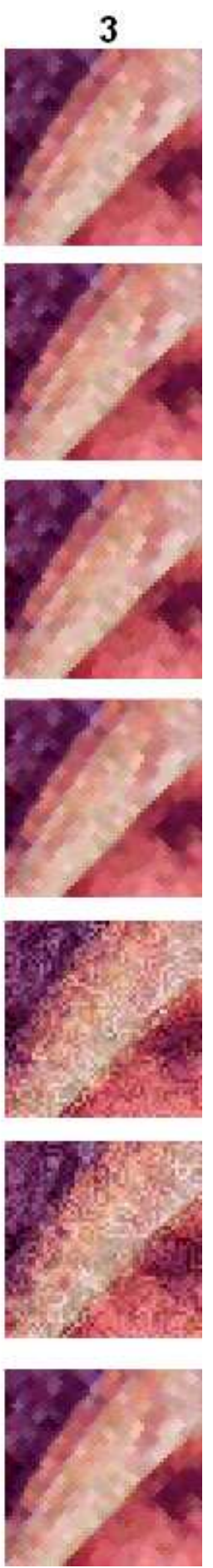

Fig. 5. Enlarged results of the area contained within the white square of figure 4, obtained on the RGB colour space against uncorrelated Gaussian noise (column 1), on the RGB colour space against correlated Gaussian noise (column 2), and on the YUV colour space against correlated Gaussian noise (column 3) 
Table 3

NMSE $\times 100$ values for the "Lenna" image obtained against highly correlated Gaussian noise

\begin{tabular}{|c||c|c|c|c|c|c|c|}
\hline \multicolumn{1}{|c||}{$\begin{array}{l}\text { Colour } \\
\text { spaces }\end{array}$} & \multicolumn{7}{|c|}{ Vector Orderings } \\
\cline { 2 - 8 } & $\mathrm{M}$ & $\mathrm{L}$ & $\mathrm{N}$ & $\mathrm{B}$ & $\mathrm{C}$ & $\mathrm{R}$ & $\mathrm{rL}$ \\
\hline RGB & $\mathbf{0 . 7 9}$ & 0.97 & 0.87 & 0.97 & 1.35 & 1.34 & 0.87 \\
\hline $\mathrm{L}^{*} \mathrm{a}^{*} \mathrm{~b}^{*}$ & $\mathbf{0 . 8 5}$ & 0.92 & 1.34 & 0.88 & 1.43 & 1.38 & 1.34 \\
\hline YUV & $\mathbf{0 . 7 9}$ & 0.89 & 0.90 & 0.88 & 1.65 & 1.67 & 0.90 \\
\hline
\end{tabular}

Table 4

Relative durations for the application of the OCCO filter on the Lenna image with a $3 \times 3$ cross shaped $\mathrm{SE}$

\begin{tabular}{|c|c|c|c|c|c|c|}
\hline $\mathrm{M}$ & $\mathrm{L}$ & $\mathrm{N}$ & $\mathrm{B}$ & $\mathrm{C}$ & $\mathrm{R}$ & $\mathrm{rL}$ \\
\hline 1.36 & 1.00 & 1.49 & 1.79 & 2.03 & 2.22 & 1.54 \\
\hline
\end{tabular}

In conclusion, the main points of this discussion include the overall superiority of the marginal approach, particularly against uncorrelated noise. On the other hand both marginal and vectorial approaches exhibit similar performances in the case of correlated noise (fig. 5). Although vectorial approaches tend to be more complex from an implementational point of view, they offer the possibility of smoothing without the risk of introducing new vectors. Additionally, the results have also underlined the importance of the colour space, as it highly influences the performance of the employed ordering.

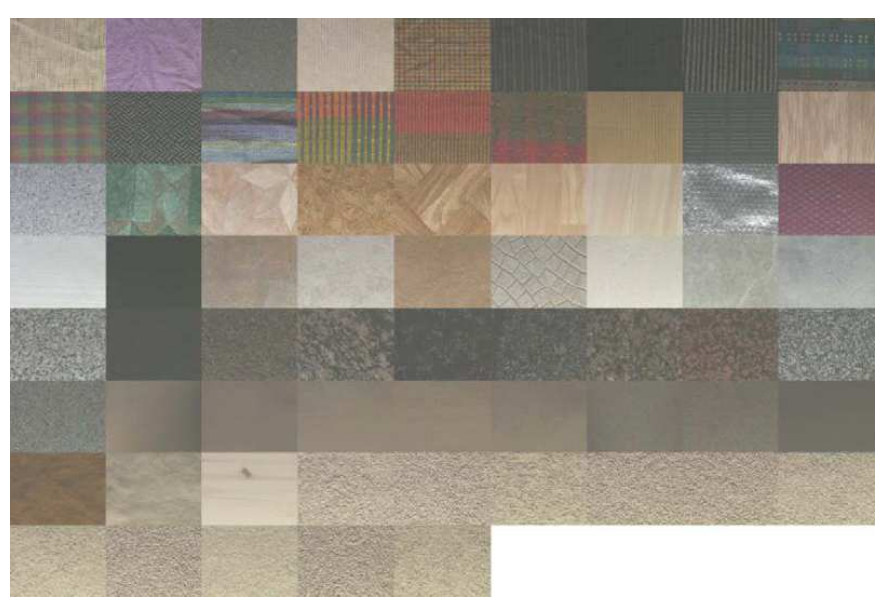

Fig. 6. Examples of the 68 textures of Outex 13 [55].

\subsection{Texture classification}

As far as texture classification is concerned, here we employ the colour textures of Outex13 (figure 6) [59]. The question whether colour should be processed 
separately or jointly from texture is still an open problem [55], and vectorial morphological feature extraction operators represent in their majority the latter case $[9,36]$. As colour texture descriptor we employ the morphological version of the autocorrelation operator, namely morphological covariance. The vectorial version of morphological covariance $K^{\prime}$ of an image $f$, is defined as the volume $\mathrm{Vol}$ of the image (i.e. sum of pixel values), eroded by a pair of points $P_{2, v}$ separated by a vector $\mathbf{v}$ :

$$
K^{\prime}\left(f ; P_{2, v}\right)=\operatorname{Vol}\left(\varepsilon_{P_{2, v}}(f)\right)
$$

In practice, $K^{\prime}$ is computed for varying lengths of $\mathbf{v}$, and most often as also here the normalised version $K$ is used for measurements:

$$
K(f)=\operatorname{Vol}\left(\varepsilon_{P_{2, v}}(f)\right) / \operatorname{Vol}(f)
$$

Given the resulting $K$ series, one can gain insight into the structure of a texture [79]. In particular, the periodic nature of covariance is strongly related to that of its input, whereas the thinness and coarseness of the textures can be evaluated by studying the sharpness of the periodic patterns within this series. Additional information concerning the anisotropy of the textures can be obtained by plotting against not only different lengths of $\mathbf{v}$, but orientations as well.

Since the SE contains only 2 pixels, the use of $\mathrm{R}$ and $\mathrm{C}$ approaches is of no practical interest. The covariance based feature vectors have been calculated using four directions for the point pairs $\left(0^{\circ}, 45^{\circ}, 90^{\circ}, 135^{\circ}\right)$, each along with distances ranging from 1 to 49 pixels in steps of size two. Consequently 25 values are available for each direction, making a total of 100 values for every image channel after concatenation. Furthermore, as far as the conversion from RGB to $\mathrm{L}^{*} \mathrm{a}^{*} \mathrm{~b}^{*}$ is concerned, the proper transformation matrix calibrated to the CIE A white point of the acquisition apparatus has been used [55]. The classification process is realised using a $\mathrm{kNN}$ classifier with $k=1$.

Table 5

Classification rates in $\%$ for the textures of Outex13, using vectorial erosion based covariance.

\begin{tabular}{|c||c|c|c|c|c|}
\hline \multicolumn{1}{|c||}{\multirow{2}{*}{$\begin{array}{c}\text { Colour } \\
\text { spaces }\end{array}$}} & \multicolumn{5}{c|}{ Vector Orderings } \\
\cline { 2 - 6 } & $\mathrm{M}$ & $\mathrm{L}$ & $\mathrm{N}$ & $\mathrm{B}$ & $\mathrm{rL}$ \\
\hline RGB & $\mathbf{7 7 . 6 5}$ & 70.74 & 71.62 & 70.86 & 71.85 \\
\hline L*a*b* $^{*}$ & 77.76 & 80.03 & 77.89 & $\mathbf{8 0 . 1 3}$ & 78.02 \\
\hline YUV & 77.66 & 79.23 & 77.75 & $\mathbf{7 9 . 4 3}$ & 77.36 \\
\hline
\end{tabular}


Table 5 contains the accuracy rates that have been obtained. In general, the relative performances can be considered similar to those obtained during noise reduction. More precisely, marginal processing continues to outperform its alternatives in RGB, once more followed by norm based ordering. Since all channels are equally important in this colour space, both prioritisation attempts with L and B fail to surpass it. However, the situation is radically modified with the other two colour spaces, where all approaches increase their performances, a result showing the effect of the colour space choice on their behaviour. Specifically, lexicographical and bitmixing orderings take the lead, though with a small margin, while they provide the overall best results in $\mathrm{L}^{*} \mathrm{a}^{*} \mathrm{~b}^{*}$.

Since luminance/luminosity alone is considered sufficient for the recognition of most image variations, its prioritisation by means of total orderings has a positive impact on the end results. Contrarily to the previous experiment however, luminosity $\left(\mathrm{L}^{*}\right)$ provides better results with respect to luminance (Y), possibly due to the use of camera specific conversion matrices. Further improvements are probably possible by decreasing the perturbations caused by the last two channels (i.e. $a^{*}, b^{*}$ and $U, V$ ), for instance by processing the last two marginally, since vector preservation is of no particular interest in this case.

\subsection{Pixel classification}

In this section we compare the different vector orderings in terms of their effect on the pertinence of features extracted from high resolution spatial data. As to the subject of the experiment, it is a multispectral image of an urban area of Strasbourg, France (fig. 7, left). It comprises mainly dense residential and commercial areas along with a few open areas as well. The image has a spatial resolution of $1.3 \mathrm{~m}$ and a size of $1100 \times 900$ pixels with 3 channels (green, red and near infra-red) common to most commercial satellites (e.g. LANDSAT, IKONOS, Quickbird, etc). Its high multispectral resolution, equivalent to the spatial resolution of panchromatic data, is due to the use of SPOT 5 sensors during a simulation flight. As a result, several small scale details and objects (e.g. cars) are present as well as long shadows, rendering the image relatively hard to classify.

Feature extraction is realised with the help of differential morphological profiles (DMP), an approach employed successfully in the field of remote sensing [65] for classifying panchromatic urban data. Its consists of a granulometry (opening profile) and of an antigranulometry (closing profile) [79]. Thus, if we extend this principle to vectorial pixels, for each $\mathbf{f}(\mathbf{x})=\left[x_{1}, x_{2}, x_{3}\right]^{T}$, two bidimensional $((n+1) \times 3)$ series are obtained, respectively the vectorial opening 
and closing profiles:

$$
\begin{aligned}
& \Pi_{\gamma}(\mathbf{f})(\mathbf{x})=\left\{\Pi_{\gamma_{\lambda}}(\mathbf{f})(\mathbf{x}) \mid \Pi_{\gamma_{\lambda}}(\mathbf{f})(\mathbf{x})=\gamma_{\lambda}(\mathbf{f})(\mathbf{x}), \forall \lambda \in\{0, \ldots, n\}\right\} \\
& \Pi_{\phi}(\mathbf{f})(\mathbf{x})=\left\{\Pi_{\phi_{\lambda}}(\mathbf{f})(\mathbf{x}) \mid \Pi_{\phi_{\lambda}}(\mathbf{f})(\mathbf{x})=\phi_{\lambda}(\mathbf{f})(\mathbf{x}), \forall \lambda \in\{0, \ldots, n\}\right\}
\end{aligned}
$$

where $\gamma_{0}(\mathbf{f})(x, y)=\phi_{0}(\mathbf{f})(x, y)=\mathbf{f}(x, y)$ and $\lambda$ denotes the size of the SE, chosen as a square of $2 \lambda+1$ pixels wide, whereas $n$ being odd represents the size of the profile. Next the derivatives of each profile are calculated by means of componentwise subtractions for every vectorial pixel. Thus the resulting multiscale feature set represents the slopes of each profile for every step of the monotonically varying SE size series.

$$
\begin{aligned}
\Delta_{\gamma}(\mathbf{f})(\mathbf{x})= & \left\{\Delta_{\gamma_{\lambda}}(\mathbf{f})(\mathbf{x}) \mid\right. \\
& \left.\Delta_{\gamma_{\lambda}}(\mathbf{f})(\mathbf{x})=\left|\Pi_{\gamma_{\lambda}}(\mathbf{f})(\mathbf{x})-\Pi_{\gamma_{\lambda-1}}(\mathbf{f})(\mathbf{x})\right|, \forall \lambda \in\{1, \ldots, n\}\right\} \\
\Delta_{\phi}(\mathbf{f})(\mathbf{x})= & \left\{\Delta_{\phi_{\lambda}}(\mathbf{f})(\mathbf{x}) \mid\right. \\
& \left.\Delta_{\phi_{\lambda}}(\mathbf{f})(\mathbf{x})=\left|\Pi_{\phi_{\lambda}}(\mathbf{f})(\mathbf{x})-\Pi_{\phi_{\lambda-1}}(\mathbf{f})(\mathbf{x})\right|, \forall \lambda \in\{1, \ldots, n\}\right\}
\end{aligned}
$$

As a last step, the result is transformed into a uni-dimensional series by separating the channel components in order to form the vectorial DMP of size $6 n$ :

$$
\begin{gathered}
\operatorname{DMP}(\mathbf{f})(\mathbf{x})=\left\{\Delta_{c}(\mathbf{f})(\mathbf{x}) \mid\right. \\
\Delta_{c}(\mathbf{f})(\mathbf{x})=\left\{\begin{array}{l}
\Delta_{\phi_{\lambda=n(1-2 i)-c+1}}^{i}(\mathbf{f})(\mathbf{x}), \forall c \in\{2 n i+1,2 n i+n\}, \forall i \in\{0,1,2\} \\
\Delta_{\gamma_{\lambda=c-n(2 i+1)}}^{i}(\mathbf{f})(\mathbf{x}), \forall c \in\{n(2 i+1)+1,2 n(1+i)\}, \forall i \in\{0,1,2\}
\end{array}\right\}
\end{gathered}
$$

where $\Delta_{\gamma_{\lambda}}^{i}$ and $\Delta_{\phi_{\lambda}}^{i}$ represent respectively the $i^{\text {th }}$ component of $\Delta_{\gamma_{\lambda}}$ and $\Delta_{\phi_{\lambda}}$. The size of the profile was fixed as $n=5$ thus providing $6 n=30$ features per vectorial pixel.

For the classification process a naive Bayes classifier is employed, which despite its extended independence assumptions, is known for its robustness. Approximately $1 \%$ of the total number of pixels is included in the training set, composed of the following five classes: buildings, roads, water, vegetation and shadows. The feature sets are extracted independently and with the exact same arguments for each ordering while the evaluation step is realised as a 10-fold cross-validation. Table 6 shows the resulting classification accuracies 

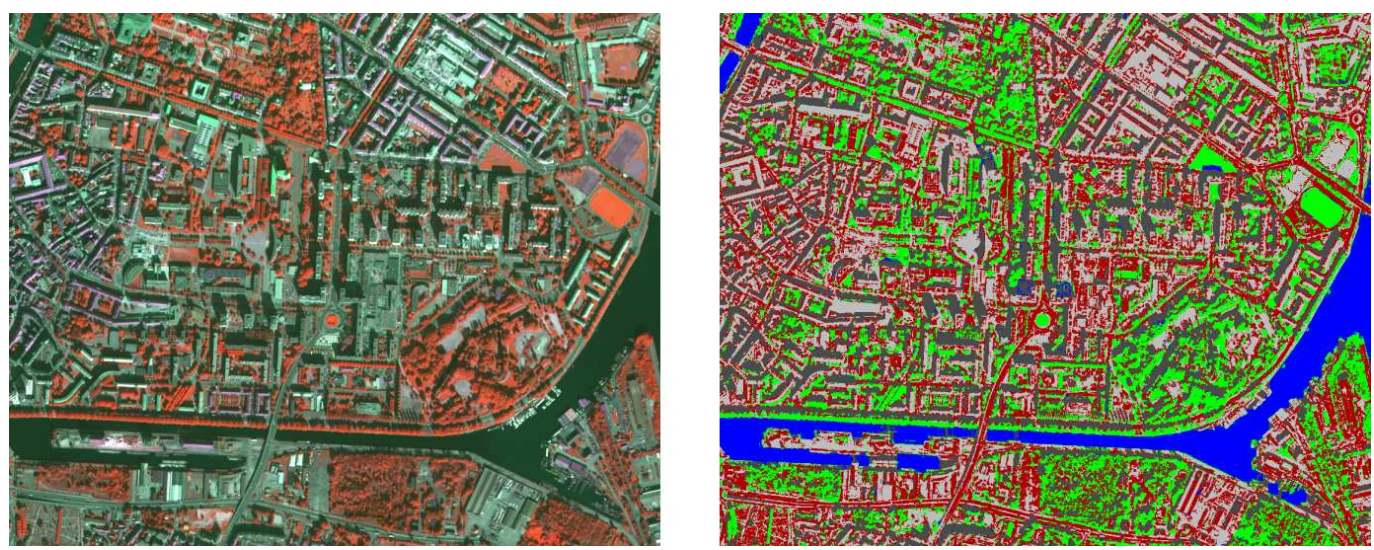

Fig. 7. The original multispectral image of Strasbourg, France in false colours (left), and the classification result using the bit mixing ordering based DMP features (right)

Table 6

Average and overall accuracies in percentage computed by means of a 10-fold cross validation

\begin{tabular}{|c||c|c|c|c|c|c|c|}
\hline \multirow{2}{*}{\multicolumn{1}{|c||}{ Class }} & \multicolumn{7}{|c|}{ Vector Orderings } \\
\cline { 2 - 8 } & $\mathrm{M}$ & $\mathrm{L}$ & $\mathrm{N}$ & $\mathrm{B}$ & $\mathrm{C}$ & $\mathrm{R}$ & $\mathrm{rL}$ \\
\hline Roads & 83.3 & 82.4 & 85.5 & 82.5 & 85.5 & 85.4 & $\mathbf{8 5 . 6}$ \\
\hline Buildings & 69.3 & 72.3 & 68.6 & $\mathbf{7 2 . 4}$ & 68.0 & 68.3 & 68.4 \\
\hline Water & 91.3 & 96.8 & 89.5 & $\mathbf{9 7 . 2}$ & 89.7 & 90.1 & 89.8 \\
\hline Vegetation & 94.0 & $\mathbf{9 4 . 4}$ & 93.1 & $\mathbf{9 4 . 4}$ & 93.0 & 92.8 & 93.4 \\
\hline Shadow & 94.2 & 92.9 & 95.1 & 92.7 & 94.7 & 94.6 & $\mathbf{9 5 . 2}$ \\
\hline \hline Average & 86.4 & $\mathbf{8 7 . 8}$ & 86.4 & $\mathbf{8 7 . 8}$ & 86.2 & 86.2 & 86.5 \\
\hline Overall & 87.0 & 88.2 & 86.9 & $\mathbf{8 8 . 3}$ & 86.8 & 86.9 & 87.0 \\
\hline
\end{tabular}

for each class; as before the entries corresponding to the best performance of their row (pixel class) are in bold.

Given the values of table 6, the overall accuracies appear to be in the magnitude of other reported uses of DMP for classification of panchromatic (monospectral) data. ( $\approx 78 \%$ for IRS-1C and $\approx 95 \%$ for IKONOS in [15]). Possible justifications of the difference include primarily the large size of the feature set. In addition, the components of the resulting vectorial features could have been combined in more sophisticated ways. Other influential factors include of course the complexity of the image as well as the efficiency of the classifier. Nevertheless, these choices were made arbitrarily because they do not bear importance in the context of this experiment as it focuses only on the relative performances of the approaches with respect to each other. 
Moreover, the overall accuracies are remarkably close ( $\mathrm{L}$ and $\mathrm{B}$ at $\approx 88 \%$ and $\mathrm{M}, \mathrm{R}, \mathrm{C}$ and $\mathrm{rL}$ at $\approx 87 \%$ ). In other words the total orderings have a slight advantage, with the exception of $\mathrm{rL}$, which once more behaves almost identically to $\mathrm{N}$ as the outcome of most comparisons is determined by the first components. The order of the channels (green, red, near infra-red) apparently works in favour of total orderings, with the chosen classes. This remark becomes more evident if we study the classification rates of each class individually, where contrarily to the overall rates, differences of more than $7 \%$ take place.

For instance, it is widely known that clear water appears dark-bluish (i.e. higher green band reflectance), which when combined with the prioritisation attributed by $\mathrm{L}$ and $\mathrm{B}$ to the first channel (i.e. green) results in more pertinent features, equivalent to visibly higher classification rates for the same two orderings with the class "water". The same logic can also be applied to the class "vegetation" which has high reflectance rates in green and in near infra-red. On the other hand, shadows, roads and buildings may appear in various shades of blue or grey, depending on their composition, hence orderings taking into account all components equally (i.e. N, C, R, M and partially $\mathrm{rL}$ ) are more effective in obtaining discriminating features, as asserted by table 6 .

In brief, despite the expectation that the restricted output of vectorial operators would damage the pertinence of the extracted features, the results show that this deficiency is compensated by their capacity to exploit the correlation of channels and thus they perform at least as well as the marginal approach. Additionally, pixel classes with distinctive spectral signatures, are more efficiently processed by orderings prioritising the corresponding channels, hence surpassing the accuracy rates of marginal ordering.

\section{Conclusion}

In this paper, we have presented an exhaustive and up to date review of the methods proposed in the literature with the end of extending the MM framework to multivalued images. We aimed especially to obtain a panorama of the existing approaches that, besides the MM community, is also accessible to those interested in multivalued image processing but not yet acquainted with the potential of multivariate MM. That is why the presentation of the theoretical foundation of the subject, that has been hardly modified since the milestone work of Goutsias et al. [33], was limited with the essential points, whereas abundant references to applications from various domains of multivalued morphological processing (i.e. colour, remote sensing, radar data, etc) were provided.

The key point of constructing morphological operators for multivalued images 
consists in defining an ordering scheme for vectorial data. Several ordering methods as well as other specialised approaches were examined in this context, with each of them equipped with their own properties (e.g. channel prioritisation, vector preservation, etc), thus rendering them suitable for certain tasks and unsuitable for others. In conclusion, there is still no single ordering scheme appropriate for all kinds of multivalued input, since their suitability is directly related to not only the overall semantic meaning of the individual channels but to the particular task under consideration as well.

The experiments that have been carried out in the second part aimed to assert the remarks concerning the theoretical properties of each ordering, and although not exhaustive, provided some interesting results. First the selected approaches have been tested in terms of noise reduction quality, where the overall superiority of the marginal processing strategy became clear, especially against uncorrelated noise, while in the case of correlated noise both marginal and vectorial orderings have produced similar smoothing rates, hence asserting the results of Comer and Delp [26]. Moreover, the same orderings have been also employed to the end of colour texture classification by means of the morphological covariance operator, where total orderings were shown to surpass their marginal counterpart in combination with a suitable colour space.

In the last experiment, multispectral remote sensing data has been used with the purpose of pixel classification. Although the so far reported cases in the literature of morphologically extracted features from this kind of images make exclusive use of the marginal approach, in this process we have showed that vector orderings chosen in relation to the properties of the desired classes hold the potential of improving the classification rates.

\section{Acknowledgements}

The authors wish to thank Prof Ch. Ronse and the anonymous reviewers for their valuable comments, as well as the members of the FoDoMuST ${ }^{1}$ project, for the remote sensing data and technical support during the classification experiments.

\section{References}

[1] H. M. Al-Otum, Morphological operators for color image processing based on Mahalanobis distance measure, Society of Photo-Optical Instrumentation

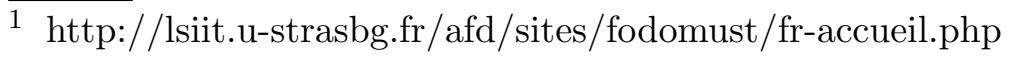


Engineers 42 (9) (2003) 2595-2606.

[2] H. M. Al-Otum, M. T. Ureikat, Color image morphology using an adaptive saturation-based technique, Optical Engineering 43 (6) (2004) 1280-1292.

[3] I. Andreadis, M. Vardavoulia, G. Louverdis, N. Papamarkos, Colour image skeletonisation, in: Proceedings of the 10th European Signal Processing Conference, vol. 4, Tampere, Finland, 2000.

[4] J. Angulo, Morphologie mathématique et indexation d'images couleur. application à la microscopie en biomédicine, Ph.D. thesis, Ecoles des Mines, Paris, France (2003).

[5] J. Angulo, Morphological color processing based on distances. Application to color denoising and enhancement by centre and contrast operators, in: Proceedings of the IASTED International Conference on Visualization, Imaging, and Image Processing (VIIP'2005), Benidorm, Spain, 2005.

[6] J. Angulo, Unified morphological color processing framework in a lum/sat/hue representation, in: C. Ronse, L. Najman, E. Decencière (eds.), Proceedings of the 7th ISMM, vol. 30 of Computational Imaging and Vision, Springer-Verlag, Dordrecht, Germany, 2005.

[7] J. Angulo, J. Serra, Mathematical morphology in color spaces applied to the analysis of cartographic images, in: S. Levachkine, J. Serra, M. Egenhofer (eds.), Proceedings of GEOPRO '03, International Workshop of Semantic Processing of Spatial Data, Mexico City, Mexico, 2003.

[8] J. Angulo, J. Serra, Morphological coding of color images by vector connected filters, in: IEEE Proceedings of the 7th International Symposium on Signal Processing and Its Applications (ISSPA'2003), vol. 1, Paris, France, 2003.

[9] E. Aptoula, S. Lefevre, Spatial morphological covariance applied to texture classification, in: Proceedings of the International Workshop on Multimedia Content Representation, Classification and Security, vol. 4105, Istanbul, Turkey, 2006 .

[10] E. Aptoula, S. Lefèvre, C. Collet, Mathematical morphology applied to the segmentation and classification of galaxies in multispectral images, in: Proceedings of the 14th European Signal Processing Conference, Florence, Italy, 2006.

[11] J. Astola, P. Haavisto, Y. Neuvo, Vector median filters, IEEE Proceedings 78 (4) (1990) 678-689.

[12] V. Barnett, The ordering of multivariate data, Journal of the Statistical Society A 139 (3) (1976) 318-355.

[13] S. Batman, J. Goutsias, Unsupervised iterative detection of land mines in highly cluttered environments, IEEE Transactions on Image Processing 12 (5) (2003) 509-523. 
[14] J. A. Benediktsson, J. A. Palmason, J. R. Sveinsson, Classification of hyperspectral data from urban areas based on extended morphological profiles, IEEE Transactions on Geoscience and Remote Sensing 43 (3) (2005) 480-491.

[15] J. A. Benediktsson, M. Pesaresi, K. Arnason, Classification and feature extraction for remote sensing images from urban areas based on morphological transformations, IEEE Transactions on Geoscience and Remote Sensing 41 (9) (2003) 1940-1949.

[16] I. Bloch, H. Maitre, Fuzzy mathematical morphologies: a comparative study, Pattern Recognition 28 (9) (1995) 1341-1387.

[17] B. Burgeth, A. Bruhn, N. Papenberg, M. Welk, J. Weickert, Mathematical morphology for matrix fields induced by the loewner ordering in higher dimensions, Signal Processing 87 (2) (2007) 277-290.

[18] B. Burgeth, N. Papenberg, A. Bruhn, M. Welk, C. Feddern, J. Weickert, Morphology for higher-dimensional tensor data via Loewner ordering, in: C. Ronse, L. Najman, E. Decencière (eds.), Proceedings of the 7th ISMM, vol. 30 of Computational Imaging and Vision, Springer-Verlag, Dordrecht, Germany, 2005, pp. 407-416.

[19] B. Burgeth, M. Welk, C. Feddern, J. Weickert, Morphological operations on matrix-valued images, in: European Conference on Computer Vision, vol. 4, Prague, Czech Republic, 2004.

[20] C. Busch, M. Eberle, Morphological operations for color-coded images, in: Proceedings of EUROGRAPHICS'95, Computer Graphics Forum, vol. 14, Maastricht, Netherlands, 1995.

[21] J. Chanussot, Approches vectorielles ou marginales pour le traitement d'images multi-composantes, Ph.D. thesis, Université de Savoie, France (1998).

[22] J. Chanussot, P. Lambert, Total ordering based on space filling curves for multivalued morphology, in: Proceedings of the International Symposium on Mathematical Morphology (ISMM'98), Amsterdam, Netherlands, 1998.

[23] J. Chanussot, P. Lambert, Watershed approaches for color image segmentation, in: Proceedings of the IEEE Workshop on nonlinear signal and image processing, Antalya, Turkey, 1999.

[24] J. Chanussot, P. Lambert, Extending mathematical morphology to color image processing, in: International Conference on Color in Graphics and Image Processing, Saint-Etienne, France, 2000.

[25] M. Comer, E. Delp, An empirical study of morphological operators in color image enhancement, in: Proceedings of the SPIE Conference on Image Processing Algorithms and Techniques III, vol. 1657, San Jose, USA, 1992.

[26] M. Comer, E. Delp, Morphological operations for color image processing, Journal of Eletronic Imaging 8 (3) (1999) 279-289. 
[27] T. Q. Deng, H. J. A. M. Heijmans, Grey-scale morphology based on fuzzy logic, Journal of Mathematical Imaging and Vision 16 (2) (2002) 155-171.

[28] M. C. d'Ornellas, R. v.d. Boomgaard, J. Geusebroek, Morphological algorithms for color images based on a generic programming approach, in: Proceedings of the Brazilian Conference on Image Processing and Computer Graphics, Rio de Janeiro, Brazil, 1998.

[29] A. N. Evans, Vector area morphology for motion field smoothing and interpretation, IEE Proceedings Vision Image and Signal Processing 150 (4) (2003) 219-226.

[30] S. Gibson, J. A. Bangham, R. Harvey, Evaluating a colour morphological scalespace, in: Proceedings of the British Machine Vision Conference, Norwhich, UK, 2003.

[31] R. C. Gonzalez, R. E. Woods, Digital Image Processing, 2nd ed., AddisonWesley, New York, 1992.

[32] J. Goutsias, H. J. A. M. Heijmans, Fundamenta morphologicae mathematicae, Fundamenta Informaticae 41 (1-2) (2000) 1-31.

[33] J. Goutsias, H. J. A. M. Heijmans, K. Sivakumar, Morphological operators for image sequences, Computer Vision and Image Understanding 62 (3) (1995) $326-346$.

[34] C. Gu, Multivalued morphology and its application in moving object segmentation and tracking, in: P. Maragos, R. W. Schafer, M. A. Butt (eds.), Mathematical Morphology and its Application to Image and Signal Processing, Kluwer Academic Publishers, Boston, USA, 1996, pp. 345-352.

[35] A. Hanbury, The morphological top-hat operator generalised to multi-channel images, in: Proceedings of the 17th International Conference on Pattern Recognition, Cambridge, UK, 2004.

[36] A. Hanbury, U. Kandaswamy, D. A. Adjeroh, Illumination-invariant morphological texture classification, in: C. Ronse, L. Najman, E. Decencière (eds.), Proceedings of the 7th ISMM, vol. 30 of Computational Imaging and Vision, Springer-Verlag, Dordrecht, Netherlands, 2005, pp. 377-386.

[37] A. Hanbury, J. Serra, Mathematical morphology in the HLS colour space, in: T. Cootes, C. Taylor (eds.), 12th British Machine Vision Conference, Manchester, UK, 2001.

[38] A. Hanbury, J. Serra, Morphological operators on the unit circle, IEEE Transactions on Image Processing 10 (12) (2001) 1842-1850.

[39] A. Hanbury, J. Serra, Mathematical morphology in the CIELAB space, Image Analysis and Stereology 21 (3) (2002) 201-206.

[40] R. C. Hardie, G. R. Arce, Ranking in $R^{p}$ and its use in multivariate image estimation, IEEE Transactions on Circuits and Systems for Video Technology 1 (2) (1991) 197-209. 
[41] H. J. A. M. Heijmans, Morphological Image Operators, Advances in Electronics and Electron Physics Series, Academic Press, Boston, 1994.

[42] H. J. A. M. Heijmans, R. Keshet, Inf-semilattice approach to self-dual morphology, Journal of Mathematical Imaging and Vision 17 (1) (2002) 5580 .

[43] H. J. A. M. Heijmans, C. Ronse, The algebraic basis of mathematical morphology, part I: dilations and erosions, Computer Vision, Graphics and Image Processing 50 (3) (1990) 245-295.

[44] R. Jones, H. Talbot, Morphological filtering for colour images with no new colours, in: Proceedings of the Image and Vision Computing New Zealand (IVCNZ'96), Lower Hutt, New Zealand, 1996.

[45] R. Keshet, Extensions of morphological operations to complete semilattices and its applications to image and video processing, in: H. Heijmans, J. Roerdink (eds.), Mathematical Morphology and its Application to Image and Signal Processing, Kluwer Academic Publishers, Dordrecht, Germany, 1998.

[46] R. Keshet, Mathematical morphology on complete semilattices and its applications to image processing, Fundamenta Informaticae $41(1 / 2)(2000)$ $33-56$.

[47] M. Köppen, C. Nowack, G. Rösel, Pareto-morphology for color image processing, in: Proceedings of the 11th Scandinavian Conference on Image Analysis, vol. 1, Kangerlussuaq, Greenland, 1999.

[48] A. Ledda, W. Philips, Majority ordering for colour mathematical morphology, in: Proceedings of the 13th European Signal Processing Conference, Antalya, Turkey, 2005.

[49] O. Lezoray, C. Meurie, A. Elmoataz, A graph approach to color mathematical morphology, in: Proceedings of the IEEE International Symposium on Signal Processing and Information Technology, Athens, Greece, 2005.

[50] J. Li, Y. Li, Multivariate mathematical morphology based on principal component analysis: initial results in building extraction, in: Proceedings of the 20th ISPRS, vol. 35, Istanbul, Turkey, 2004.

[51] G. Louverdis, I. Andreadis, P. Tsalides, Morphological granulometries for color images, in: 2nd Hellenic Conference on Artificial Intelligence, Thessaloniki, Greece, 2002.

[52] G. Louverdis, I. Andreadis, P. Tsalides, A new fuzzy model for morphological colour image processing, IEE Proceedings - Vision, Image and Signal Processing 149 (3) (2002) 129-139.

[53] G. Louverdis, M. Vardavoulia, I. Andreadis, P. Tsalides, A new approach to morphological color image processing, Pattern Recognition 35 (8) (2002) 17331741. 
[54] R. Lukac, B. Smolka, K. Martin, K. N. Plataniotis, A. N. Venetsanopoulos, Vector filtering for color imaging, IEEE Signal Processing Magazine 22 (1) (2005) 74-86.

[55] T. Mäenpää, M. Pietikäinen, Classification with color and texture: jointly or separately?, Pattern Recognition 37 (8) (2004) 1629-1640.

[56] K. V. Mardia, J. Kent, J. Bibby, Multivariate Analysis, Academic Press, London, 1976.

[57] G. Matheron, Random sets and integral geometry, Wiley, New York, 1975.

[58] A. Mojsilovic, E. Soljanin, Quantization of color spaces and processing of color images by fibonacci lattices, in: Proceedings of Human Vision and Electronic Imaging V, vol. SPIE 3959, San Jose, USA, 2000.

[59] T. Ojala, T. Mäenpää, M. Pietikäinen, J. Viertola, J. Kyllönen, S. Huovinen, Outex: New framework for empirical evaluation of texture analysis algorithms, in: Proceedings of the 16th ICPR, vol. 1, Quebec City, Canada, 2002.

[60] F. Ortiz, Colour mathematical morphology for neural image analysis, Real-Time Imaging 8 (5) (2002) 455-465.

[61] F. Ortiz, F. Torres, Vectorial morphological reconstruction for brightness elimination in colour images, Real-Time Imaging 10 (6) (2004) 379-387.

[62] F. Ortiz, F. Torres, J. Angulo, S. Puente, Comparative study of vectorial morphological operations in different color spaces, Proceedings of Intelligent robots and computer vision XX: Algorithms, Techniques, and Active Vision 4572 (2001) 259-268.

[63] F. Ortiz, F. Torres, P. Gil, Gaussian noise elimination in colour images by vector-connected filters, in: Proceedings of the 17th International Conference on Pattern Recognition, vol. 4, Cambridge, UK, 2004.

[64] F. Ortiz, F. Torres, P. Gil, J. Pomares, S. Puente, F. Candelas, Ordering by distance for hsi mathematical morphology, in: Proceedings of the 9th Spanish Symposium on Pattern Recognition and Image Analysis, vol. 2, Castellón, Spain, 2001.

[65] M. Pesaresi, J. Benediktsson, A new approach for the morphological segmentation of high resolution satellite imagery, IEEE Transactions on Geoscience and Remote Sensing 39 (2) (2001) 309-320.

[66] A. Peters, Mathematical morphology for angle-valued images, in: Proceedings of SPIE, Nonlinear Image Processing VIII, vol. 3026, 1997.

[67] I. Pitas, P. Tsakalides, Multivariate ordering in color image filtering, IEEE Transactions on Circuits and Systems for Video Technology 1 (3) (1991) 247259.

[68] A. Plaza, P. Martinez, R. Perez, J. Plaza, A new approach to mixed pixel classification of hyperspectral imagery based on extended morphological profiles, Pattern Recognition 37 (6) (2004) 1097-1116. 
[69] J. Rivest, Morphological operators on complex signals, Signal Processing 84 (1) (2004) 133-139.

[70] J. Rivest, Granulometries and pattern spectra for radar signals, Signal Processing 86 (5) (2006) 1094-1103.

[71] C. Ronse, Why mathematical morphology needs complete lattices, Signal Processing 21 (2) (1990) 129-154.

[72] C. Ronse, V. Agnus, Morphology on label images: Flat-type operators and connections, Journal of Mathematical Imaging and Vision 22 (2/3) (2005) 283307.

[73] C. Ronse, H. J. A. M. Heijmans, The algebraic basis of mathematical morphology, part II: openings and closings, Computer Vision, Graphics and Image Processing 54 (1) (1991) 74-97.

[74] L. J. Sartor, A. R. Weeks, Morphological operations on color images, Journal of Electronic Imaging 10 (2) (2001) 548-559.

[75] J. Serra, Image Analysis and Mathematical Morphology Vol I, Academic Press, London, 1982.

[76] J. Serra, Image Analysis and Mathematical Morphology Vol II: Theoretical Advances, Academic Press, London, 1988.

[77] J. Serra, Anamorphoses and function lattices, in: E. R. Dougherty (ed.), Mathematical Morphology in Image Processing, chap. 13, Marcel Dekker, New York, 1993, pp. 483-523.

[78] P. Soille, Applications of morphological operators, in: B. Jähne, H. Haußecker, P. Geißler (eds.), Handbook of Computer Vision and Applications, vol. 3, chap. 12, Academic Press, San Diego, 1999, pp. 283-296.

[79] P. Soille, Morphological Image Analysis : Principles and Applications, SpringerVerlag, Berlin, 2003.

[80] P. Soille, M. Pesaresi, Advances in mathematical morphology applied to geoscience and remote sensing, IEEE Transactions on Geoscience and Remote Sensing 40 (9) (2002) 2042-2055.

[81] E. Stringa, A. Teschioni, C. S. Regazzoni, A classical morphological approach to color image filtering based on space filling surves, in: Proceedings of the IEEE-Eurasip Workshop on Nonlinear Signal and Image Processing, Antalya, Turkey, 1999.

[82] H. Talbot, C. Evans, R. Jones, Complete ordering and multivariate mathematical morphology, in: H. Heijmans, J. Roerdink (eds.), Mathematical Morphology and Its Applications to Image and Signal Processing, Kluwer Academic Press, Amsterdam, 1998.

[83] K. Tang, J. Astola, Y. Neuvo, Multivariate ordering and filtering with application to color image processing, in: Proceedings of the 6th European Signal Processing Conference, Brussels, Belgium, 1992. 
[84] D. M. Titterington, Estimation of correlation coefficients by ellipsoid trimming, Applied Statistics 27 (3) (1978) 227-234.

[85] A. Toet, Detection of dim point targets in cluttered maritime backgrounds through multisensor image fusion, in: W. R. Watkins, D. Clement, W. R. Reynolds (eds.), SPIE Conference, Targets and Backgrounds VIII: Characterization and Representation, vol. 4718, Orlando, USA, 2002.

[86] P. E. Trahanias, A. N. Venetsanopoulos, Color edge detection based on multivariate ordering, in: SPIE Conference, Visual Communications and Image Processing, Boston, USA, 1992.

[87] P. E. Trahanias, A. N. Venetsanopoulos, Multichannel image processing using vector-angle ranking, in: E. R. Dougherty, J. Astola, H. G. Longbotham (eds.), SPIE Conference, Nonlinear Image Processing IV, vol. 1902, San Jose, USA, 1993.

[88] P. E. Trahanias, A. N. Venetsanopoulos, Vector directional filters - a new class of multichannel image processing filters, IEEE Transactions on Image Processing 2 (4) (1993) 528-534.

[89] A. Trémeau, C. Fernandez-Maloigne, P. Bonton, Image Numérique Couleur, Dunod, Paris, 2004.

[90] M. T. Ureikat, H. M. Al-Otum, Angle-valued Mahalanobis-based image morphological operators, in: Proceedings of the 4th WSEAS International Conference on Signal, Speech and Image Processing, Izmir, Turkey, 2004.

[91] M. I. Vardavoulia, I. Andreadis, P. Tsalides, A new vector median filter for colour image processing, Pattern Recognition Letters 22 (6-7) (2001) 675-689.

[92] M. I. Vardavoulia, I. Andreadis, P. Tsalides, Vector ordering and morphological operations for colour image processing: fundamentals and applications, Pattern Analysis and Applications 5 (3) (2002) 271-287.

[93] C. Vertan, M. Malciu, V. Buzuloiu, V. Popescu, Median filtering techniques for vector valued signals, in: Proceedings of the 3rd IEEE International Conference on Image Processing, vol. 1, Lausanne, Switzerland, 1996.

[94] C. Vertan, V. Popescu, V. Buzuloiu, Morphological like operators for color images, in: Proceedings of the 8th European Signal Processing Conference, Trieste, Italy, 1996.

[95] K. R. Weber, S. T. Acton, On connected filters in color image processing, Journal of Electronic Imaging 13 (3) (2004) 619-629.

[96] M. Wheeler, M. A. Zmuda, Processing color and complex data using mathematical morphology, in: IEEE Proceedings of the National Aerospace and Electronics Conference, vol. 1, Dayton, USA, 2000.

[97] S. S. Wilson, Theory of matrix morphology, IEEE Transactions on Pattern Analysis and Machine Intelligence 14 (6) (1992) 636-652. 
[98] E. Zaharescu, M. Zamfir, C. Vertan, Color morphology-like operators based on color geometric shape characteristics, in: Proceedings of the International Symposium on Signals, Circuits and Systems, vol. 1, Bucarest, Romania, 2003. 